\title{
First-principles investigation of Ag-Cu alloy surfaces in an oxidizing environment
}

\author{
Simone Piccinin and Catherine Stampfl \\ School of Physics, The University of Sydney, Sydney, New South Wales 2006, Australia \\ Matthias Scheffler \\ Fritz-Haber-Institut der Max-Planck-Gesellschaft, Faradayweg 4-6, D-14195 Berlin, Germany
}

(Received 25 October 2007; published 26 February 2008)

\begin{abstract}
In this paper, we investigate by means of first-principles density functional theory calculations the (111) surface of the $\mathrm{Ag}-\mathrm{Cu}$ alloy under varying conditions of pressure of the surrounding oxygen atmosphere and temperature. This alloy has been recently proposed as a catalyst with improved selectivity for ethylene epoxidation with respect to pure silver, the catalyst commonly used in industrial applications. Here, we show that the presence of oxygen leads to copper segregation to the surface. Considering the surface free energy as a function of the surface composition, we construct the convex hull to investigate the stability of various surface structures. By including the dependence of the free surface energy on the oxygen chemical potential, we are able compute the phase diagram of the alloy as a function of temperature, pressure, and surface composition. We find that, at temperature and pressure, typically used in ethylene epoxidation, a number of structures can be present on the surface of the alloy, including clean $\operatorname{Ag}(111)$, thin layers of copper oxide, and thick oxidelike structures. These results are consistent with, and help explain, recent experimental results.
\end{abstract}

DOI: 10.1103/PhysRevB.77.075426

PACS number(s): 68.43.Bc, 81.65.Mq, 61.82.Bg

\section{INTRODUCTION}

Silver is the active element in heterogeneous catalysis for a number of industrially important chemical reactions including ethylene epoxidation (carried out at atmospheric pressure and temperatures $T=500-600 \mathrm{~K}$ ) (Ref. 1) and partial oxidation of methanol to formaldehyde (conducted at atmospheric pressure and $T=800-900 \mathrm{~K}$ ). The origin of the high catalytic activity of such a noble metal has been attributed to the thin oxidelike structures present on the lowest energy surface for realistic operating conditions. ${ }^{2-4}$ In agreement with the Sabatier principle (in which a good catalyst readily dissociates adparticles but does not bind the fragments too strongly), it has been argued that while clean $\operatorname{Ag}(111)$ binds the adsorbates too weakly, the thin oxides provide $\mathrm{O}-\mathrm{Ag}$ bonds of intermediate strength. A number of energetically very similar oxidelike structures have been identified for this system, ${ }^{3}$ suggesting a scenario in which the surface might comprise of not simply a single low energy structure, but could well be dynamically evolving in time, fluctuating between different structures. This picture also emerges from recent studies on $\mathrm{CO}$ oxidation at $\mathrm{RuO}_{2}(110)$ (Ref. 5) and $\operatorname{Pd}(100) .{ }^{6}$ In both cases, the highest catalytic activity is reached in regions of the phase diagram corresponding to boundaries between different stable surface oxide structures. Experiments conducted under semirealistic catalytic conditions for $\mathrm{CO}$ oxidation at $\mathrm{Pt}(110)$ and $\mathrm{Pd}(100)$ (Ref. 7) have also shown how, under steady-state catalysis, the thin oxides present at the surface continuously evolve with time. These recent results have therefore stressed how in catalysis, a nonequilibrium process, the catalyst surface cannot be viewed as a static object but rather as a "living" system that, at the atomistic level, continuously evolves due to various processes such as adsorption, desorption, association, dissociation, and diffusion.

Having a detailed knowledge of the catalyst surface structures under operating conditions is therefore a crucial first step toward understanding the full catalytic process at the atomistic level. Conventional surface science investigations are conducted in ultrahigh vacuum (UHV) and at room temperature or below, while industrial applications of heterogeneous catalysis usually require pressure of the order of atmospheres and temperatures often higher than room temperature. The information extracted from such surface science experiments cannot always be extrapolated to realistic conditions since structures that can exist at high temperature and pressure might not be seen in UHV conditions and vice versa. Bridging this difference in ambient conditions (usually referred to as the pressure and temperature gap) is one of the major goals in current surface science research. To this end, first-principles simulations that combine accurate electronic structure methods with equilibrium thermodynamics have been successfully employed to predict the stability of surface structures of several systems under high temperature and high pressure conditions. ${ }^{8}$ Moreover, such methods have been included in a multiscale approach that employs statistical mechanics methods to simulate the full steadystate catalysis cycle. In some cases, the agreement with experiments can be quantitative, allowing an unprecedented level of insight into the concerted actions of a dynamical process such as heterogeneous catalysis. ${ }^{5}$

With regard to the silver catalyst, the mechanism of ethylene epoxidation has been studied in two recent works ${ }^{9,10}$ using a combination of experimental and theoretical techniques. It has been proposed that both the selective (producing ethylene oxide) and unselective (producing the acetaldehyde intermediate and finally leading to total oxidation) reaction pathways have a common intermediate, a surface oxametallacycle. This intermediate can react to form either ethylene oxide or acetaldehyde with similar activation barriers. The same authors also reported, on the basis of both first-principles calculations ${ }^{11}$ and experiments, ${ }^{12}$ that if an $\mathrm{Ag}-\mathrm{Cu}$ alloy, rather than pure $\mathrm{Ag}$, is used as a catalyst, the selectivity toward ethylene oxide will be improved. 
The use of bimetallic catalysts such as $\mathrm{Ag}-\mathrm{Cu}$ has been the focus of much work in the field of heterogeneous catalysis ${ }^{13}$ since the catalytic activity and selectivity of a metal can be modified substantially by alloying with another metal. For example, geometrical and electronic effects obtained by varying the alloy composition may play a crucial role in determining the properties of the catalyst. ${ }^{14}$ One appealing aspect of bimetallic alloys is the possibility of rationally designing the catalytic properties of the material by changing the alloy elements and composition. To this end, first-principles calculations have been shown to be a potentially useful tool for screening among pools of possible alloys and for extracting trends and therefore gaining insights into the functioning of the alloy. ${ }^{15,16}$ In view of the above discussion, though, the effects of high pressure and temperature and the role of the dynamical evolution of the catalyst must be carefully accounted for, in order for the theoretical simulations to have predictive power.

Through ex situ x-ray photoelectron spectroscopy (XPS) measurements of $\mathrm{Ag}-\mathrm{Cu}$ catalysts, it has been shown that the copper surface content is much higher than the overall copper content of the alloy, ${ }^{11}$ therefore suggesting copper segregation to the surface. This lead Linic and Barteau ${ }^{9,10}$ to theoretically model the surface of the $\mathrm{Ag}-\mathrm{Cu}$ alloy assuming a perfect $\mathrm{Ag}(111)$ surface in which one out of four silver atoms is replaced by a copper atom. However, it is known that copper, at the temperature $T$ and pressure $p_{\mathrm{O}_{2}}$ used in such experiments $\left(T=528 \mathrm{~K}, p_{\mathrm{O}_{2}}=0.1 \mathrm{~atm}\right)$, oxidizes to $\mathrm{CuO},{ }^{17}$ while at higher temperature or lower pressure, $\mathrm{Cu}_{2} \mathrm{O}$ is the stable oxide. ${ }^{17}$ Thus, it is possible that more complex structures involving copper oxide can be present on the catalyst surface.

The aim of this paper is to investigate, by means of firstprinciples electronic structure calculations, the surface structure of such alloys under varying oxygen pressure and temperature conditions. To do this, we consider the alloy surface to be in thermodynamic equilibrium with an atmosphere of pure oxygen. The effect of the presence of other reactants such as ethylene is not investigated in this work. Under the conditions of steady-state catalysis, it is therefore likely that the surface of the catalyst might be modified with respect to the stable structures found in an oxygen atmosphere. Hence, our work can be thought of as a first step in gaining some insight in the possible structures of the alloy, an essential prerequisite for modeling the full catalytic process.

The paper is organized as follows. In Sec. II, we briefly review the theoretical background relevant for this work, present the definitions of the quantities that will be used throughout the paper, and discuss the approximations and assumptions that underpin our methodology. In Sec. III, we report our results and discuss their relevance. Section IV summarizes the main findings.

\section{CALCULATION METHOD}

The density functional theory (DFT) calculations presented in this work are performed using the generalized gradient approximation (GGA) of Perdew-Burke-Ernzerhof ${ }^{18}$ (PBE) for the exchange and correlation functional. We use ultrasoft pseudopotentials ${ }^{19,20}$ for the electron-ion interactions, including scalar relativistic effects. The Kohn-Sham wave functions are expanded in plane waves with an energy cutoff of 27 Ry (200 Ry for the charge density cutoff). To sample the Brillouin zone, we use the special-point technique, ${ }^{21}$ broadening the Fermi surface according to the Marzari-Vanderbilt cold-smearing technique ${ }^{22}$ using a smearing parameter of $0.03 \mathrm{Ry}$. In the $(1 \times 1)$ surface unit cell, corresponding to the periodicity of clean $\mathrm{Ag}(111)$, a $12 \times 12 \times 1$ k-point mesh is used: This amounts to $19 \mathbf{k}$ points in the irreducible part of the Brillouin zone and gives adsorption energies for oxygen adsorption converged to within $16 \mathrm{meV}$ (Ref. 23) at full coverage. For larger unit cells, the k-point mesh is scaled accordingly. All the calculations are performed using the PWSCF code contained in the QUANTUM-ESPRESSO package. ${ }^{24}$ For the calculations of chemisorbed structures, we employ a seven layer symmetric slab geometry, with adsorbates on both sides of the slab and fixing the position of the three central layers. To model oxidelike structures, we place the thin copper layers on one side of a three layer Ag slab, fixing the bottom two layers. ${ }^{25}$ The positions of the other atoms are relaxed until the forces are less than $0.001 \mathrm{Ry} / \mathrm{au}(0.025 \mathrm{eV} / \AA)$. A $12 \AA$ vacuum layer is used, which is found to be sufficient to ensure negligible coupling between periodic replicas of the slab. ${ }^{26}$ For the inplane lattice spacing, we use the calculated equilibrium bulk fcc lattice parameter of $\mathrm{Ag}, a_{0}=4.16 \AA$. The experimental value for the lattice parameter is $4.09 \AA .^{27}$

We now define some quantities that will help the discussion presented in the following sections. In the case of adsorption of oxygen on a clean $\mathrm{Ag}$ surface, the average binding energy per oxygen atom is defined as

$$
E_{b}^{\mathrm{O} / \mathrm{Ag}}=-\frac{1}{N_{\mathrm{O}}}\left[E^{\mathrm{O} / \mathrm{Ag}}-\left(E^{\mathrm{slab}}+N_{\mathrm{O}} E^{\mathrm{O}}\right)\right],
$$

where $N_{\mathrm{O}}$ is the number of oxygen atoms in the unit cell, and $E^{\mathrm{O} / \mathrm{Ag}}, E^{\mathrm{S} \text { lab }}$, and $E^{\mathrm{O}}$ are the energies of the total system, the Ag slab, and half the oxygen molecule (which has been computed through a spin-polarized DFT calculation), i.e., $E^{\mathrm{O}}=1 / 2 E_{\mathrm{O}_{2}}^{\mathrm{total}}$. Defined this way, a positive binding energy means that the adsorption of an oxygen atom is exothermic (i.e., stable) with respect to oxygen in molecular gas phase form. We point out here that the significant error $(\sim 0.54 \mathrm{eV})$ in the oxygen molecule binding energy introduced by the GGA-PBE approximation $\left[1 / 2 E_{b}^{\mathrm{O}_{2}}=3.10 \mathrm{eV}\right.$, while the experimental value is $2.56 \mathrm{eV}$ (Ref. 28)] would introduce a large error bar in the determination of the oxygen atom energy and therefore in the oxygen atom chemical potential. This error, however, will partially be compensated by the analogous GGA-PBE error in the description of oxygen chemisorbed on the metal surfaces.

To take into account the effects of temperature $(T)$ and pressure $(p)$, we employ ab initio atomistic thermodynamics, ${ }^{29-31}$ which allows the determination of the lowest energy structures as a function of the $T$ and $p$. The surface is considered to be in contact with an oxygen atmosphere that acts as a reservoir, therefore exchanging oxygen 
atoms with the surface without changing its temperature and pressure (i.e., its chemical potential).

The change in Gibbs free energy is calculated as

$$
\Delta G\left(\mu_{\mathrm{O}}\right)=\frac{1}{A}\left(G^{\mathrm{O} / \mathrm{Ag}}-G^{\text {slab }}-\Delta N_{\mathrm{Ag}} \mu_{\mathrm{Ag}}-N_{\mathrm{O}} \mu_{\mathrm{O}}\right),
$$

where $G^{\mathrm{O} / \mathrm{Ag}}$ is the free energy of the adsorbate and/or substrate structure, $G^{\text {slab }}$ is the free energy of the clean Ag slab, $\Delta N_{\mathrm{Ag}}$ is the difference in the number of $\mathrm{Ag}$ atoms between the adsorption system and the clean Ag slab, and $\mu_{\mathrm{Ag}}$ and $\mu_{\mathrm{O}}$ are the atom chemical potentials of $\mathrm{Ag}$ and $\mathrm{O}$. The change in Gibbs free energy is normalized by the surface area $A$ to allow comparisons between structures with different unit cells; we will refer to this quantity as "change in Gibbs surface free energy of adsorption," or simply "surface free energy." The chemical potential of Ag is taken to be that of an $\mathrm{Ag}$ atom in bulk $\mathrm{Ag}$, therefore assuming that the slab is in equilibrium with the bulk that acts as the silver reservoir. The oxygen chemical potential depends on temperature and pressure according to ${ }^{32}$

$$
\mu_{\mathrm{O}}(T, p)=\frac{1}{2}\left[E_{\mathrm{O}_{2}}^{\mathrm{total}}\left(T, p^{0}\right)+\tilde{\mu}_{\mathrm{O}_{2}}\left(T, p^{0}\right)+k_{B} T \ln \left(\frac{p_{\mathrm{O}_{2}}}{p^{0}}\right)\right] .
$$

Here, $p^{0}$ is the standard pressure and $\widetilde{\mu}_{\mathrm{O}_{2}}\left(T, p^{0}\right)$ is the chemical potential at the standard pressure, which can obtained either from thermochemical tables ${ }^{33}$ (the choice made in this study) or directly computed.

The vibrational contributions to the free energy, which in principle should be accounted for, have been shown to be sufficiently small so as not to play an important role for the $\mathrm{O} / \mathrm{Ag}$ system. ${ }^{30,31}$ When comparing systems with different stoichiometries, on the other hand, vibrational contributions might play a non-negligible role. In this work, however, we will neglect such effects. To show that this approximation is valid, we follow the procedure described in Ref. 32 to estimate the vibrational contribution to the surface free energy using the Einstein model and approximating the phonon density of states by just one characteristic frequency for oxygen. We find that for two of the most stable structures ("p2" and "CuO(1L)," see Sec. III), the oxygen characteristic frequencies are 62 and $69 \mathrm{meV}$, respectively, giving rise to differences in free surface energy of less than $10 \mathrm{meV} / \AA^{2}$ in the range of temperatures of interest $(0-1000 \mathrm{~K})$. This estimate indicates that such contributions are indeed small and that neglecting these terms will not alter the main conclusions of our paper. Hence, within this model, the only term that depends on $T$ and $p$ is the oxygen chemical potential. As a consequence, the free energies $G^{\mathrm{O} / \mathrm{Ag}}$ and $G^{\text {slab }}$ are identified as the total energies $E^{\mathrm{O} / \mathrm{Ag}}$ and $E^{\text {slab }}$.

If we now consider the presence of copper impurities in the silver surface, we need to modify the definition of surface free energy. As we will show later in the paper, we will deal with structures in which an overlayer containing $\mathrm{O}, \mathrm{Ag}$, and $\mathrm{Cu}$ is adsorbed on a clean $\mathrm{Ag}$ surface. If we consider $\mathrm{Cu}$ to be in equilibrium with a bulk $\mathrm{Cu}$ reservoir, the definition of surface free energy becomes
$\Delta G\left(\mu_{\mathrm{O}}\right)=\frac{1}{A}\left(G^{\mathrm{O} / \mathrm{Cu} / \mathrm{Ag}}-G^{\mathrm{slab}}-\Delta N_{\mathrm{Ag}} \mu_{\mathrm{Ag}}-N_{\mathrm{Cu}} \mu_{\mathrm{Cu}}-N_{\mathrm{O}} \mu_{\mathrm{O}}\right)$,

where $N_{\mathrm{Cu}}$ is the number of $\mathrm{Cu}$ atoms and $\mu_{\mathrm{Cu}}$ the copper chemical potential. Since in this work, as we will show in Sec. III, we are interested in identifying the structures belonging to the convex hull of the free energy vs copper content curve, which depends on the curvature of such curve, the choice of the $\mathrm{Cu}$ chemical potential is arbitrary, since the surface free energy depends linearly on it [see Eq. (4)]. We now define the average oxygen binding energy in the whole structure as

$$
\begin{aligned}
E_{b}^{\mathrm{O} / \mathrm{Cu} / \mathrm{Ag}}= & -\frac{1}{N_{\mathrm{O}}}\left[E^{\mathrm{O} / \mathrm{Cu} / \mathrm{Ag}}-\left(E^{\mathrm{slab}}+\Delta N_{\mathrm{Ag}} \mu_{\mathrm{Ag}}\right.\right. \\
& \left.\left.+N_{\mathrm{Cu}} \mu_{\mathrm{Cu}}+N_{\mathrm{O}} E^{\mathrm{O}}\right)\right] .
\end{aligned}
$$

Using the above-mentioned approximations, we can express the change in Gibbs surface free energy in terms of the average oxygen binding energy,

$$
\Delta G\left(\Delta \mu_{\mathrm{O}}\right)=-\frac{1}{A}\left(N_{\mathrm{O}} E_{b}^{\mathrm{O} / \mathrm{Cu} / \mathrm{Ag}}+N_{\mathrm{O}} \Delta \mu_{\mathrm{O}}\right),
$$

where the oxygen chemical potential is now measured with respect to half an isolated $\mathrm{O}_{2}$ molecule: $\Delta \mu_{\mathrm{O}}=\mu_{\mathrm{O}}-\frac{1}{2} E_{\mathrm{O}_{2}}^{\text {total }}$.

In writing the change in free energy as in Eq. (6), we assume that the configurational entropy contribution to the free energy is negligible. To show that this is indeed the case, we compute the mixing entropy, ${ }^{34}$

$$
S^{\text {mix }}=-k_{B}\left[x_{s}^{\alpha} \ln \left(x_{s}^{\alpha}\right)+\left(1-x_{s}^{\alpha}\right) \ln \left(1-x_{s}^{\alpha}\right)\right],
$$

where $x_{s}^{\alpha}$ is the concentration of the disordered phase $\alpha$ and $k_{B}$ is the Boltzmann constant. The quantity $-T S^{\text {mix }}$ around temperatures of interest for catalysis $(T=600 \mathrm{~K})$ has a maximum for $x_{s}^{\alpha}=0.5$, where it takes a value of $5 \mathrm{meV} / \AA^{2}$. As we will show later, such contribution is negligible compared to the energy scale of the surface free energies of the structures considered in this work.

\section{RESULTS}

\section{A. Copper segregation to the surface}

Copper and silver are known to be almost completely immiscible in the bulk due to their large size mismatch (13\%): The maximum solubility limit is 8.2 mass $\% \mathrm{Cu}$ at the eutectic temperature of $779{ }^{\circ} \mathrm{C}$ and rapidly decreases at lower temperatures, with a value of 0.7 mass $\% \mathrm{Cu}$ at $400{ }^{\circ} \mathrm{C} .{ }^{35}$ Accordingly, a DFT calculation at $0 \mathrm{~K}$ shows that the alloy mixing energy, defined as

$$
\sigma^{\text {mix }}=\left(E^{\text {total }}-N_{\mathrm{Ag}} \mu_{\mathrm{Ag}}-N_{\mathrm{Cu}} \mu_{\mathrm{Cu}}\right) /\left(N_{\mathrm{Ag}}+N_{\mathrm{Cu}}\right),
$$

where $E^{\text {total }}$ is the total energy of the unit cell of the alloy structure and is positive for all the concentrations considered here (between 1.4\% and 6.2\%). The results are shown in Table I. Extrapolating the results at zero $\mathrm{Cu}$ concentration gives a positive mixing energy, therefore inhibiting alloying 
TABLE I. Alloy mixing energy $\sigma^{\text {mix }}$ as a function of the concentration of $\mathrm{Cu}$ impurities (in percent) in bulk Ag. The simulation cells used are indicated in the first column, given in terms of the conventional unit cell vectors, which have been kept fixed at the equilibrium $\mathrm{Ag}$ lattice constant. $N_{\text {tot }}$ is the total number of atoms and $N_{\mathrm{Cu}}$ is the number of copper atoms present in the simulation cell.

\begin{tabular}{lcccc}
\hline \hline Cell & $\% \mathrm{Cu}$ & $N_{\mathrm{Cu}}$ & $N_{\text {tot }}$ & $\begin{array}{c}\sigma^{\text {mix }} \\
(\mathrm{meV} / \text { atom })\end{array}$ \\
\hline $2 \times 2 \times 1$ & 6.2 & 1 & 16 & 18.4 \\
$2 \times 2 \times 3$ & 4.2 & 2 & 48 & 12.6 \\
$2 \times 2 \times 2$ & 3.1 & 1 & 32 & 9.5 \\
$2 \times 2 \times 3$ & 2.1 & 1 & 48 & 6.5 \\
$2 \times 3 \times 3$ & 1.4 & 1 & 72 & 4.4 \\
\hline \hline
\end{tabular}

at $0 \mathrm{~K}$ at all concentrations. Here, we relax all the atomic positions and keep the simulation cell fixed and commensurate to the computed equilibrium Ag lattice constant. One would therefore expect $\mathrm{Ag}$ and $\mathrm{Cu}$ to phase segregate at low temperatures, where entropic effects are negligible.

If rather than bulk solid solutions we consider the presence of surfaces, we have to note that the surface energy of the most stable surfaces of $\mathrm{Ag}$ and $\mathrm{Cu}$ differ significantly: The computed surface energy of $\operatorname{Ag}(111), \quad \gamma^{\operatorname{Ag}(111)}$ $=0.047 \mathrm{eV} / \AA^{2}$ (expt., $\left.{ }^{36} 0.078 \mathrm{eV} / \AA^{2}\right)$, is much smaller than that of $\mathrm{Cu}(111), \quad \gamma^{\mathrm{Cu}(111)}=0.076 \mathrm{eV} / \AA^{2} \quad$ (expt., ${ }^{37}$ $0.114 \mathrm{eV} / \AA^{2}$ ).

This suggests that, when copper impurities are introduced in silver, it is unlikely for copper to be exposed on the surface. To verify this, we compute the total energies of $\mathrm{Ag}$ slabs in which one substitutional copper atom is positioned in different layers, and all the atomic coordinates are fully relaxed, with the in-plane dimensions of the simulation cell fixed to the Ag lattice constant. We find that both bulk and surface positions are unfavored compared to the position directly below the first layer. These results are summarized in Table II. We can see that by increasing the size of the surface cell the qualitative picture is unchanged. In Fig. 1, we show the unit cell used in the case of the $(3 \times 3)$ cell.

Accordingly, we also find that increasing the content of $\mathrm{Cu}$ in the first layer of the slab leads to an increase of the

TABLE II. Difference in total energy between the reference structure (where $\mathrm{Cu}$ occupies a $\mathrm{Ag}$ site in the center of the seven layer slab, i.e., in layer 4) and with the $\mathrm{Cu}$ impurity positioned in other layers. The Ag layer in which $\mathrm{Cu}$ sits is indicated in the first column. The $(1 \times 1),(2 \times 2)$, and $(3 \times 3)$ surface unit cells have been considered.

\begin{tabular}{lccc}
\hline \hline & \multicolumn{3}{c}{$\Delta E(\mathrm{meV})$} \\
\cline { 2 - 4 } Ag @ layer & $(1 \times 1)$ & $(2 \times 2)$ & $(3 \times 3)$ \\
\hline 1 & 56 & 93 & 76 \\
2 & -200 & -68 & -74 \\
3 & -103 & -38 & -39 \\
4 & 0 & 0 & 0 \\
\hline \hline
\end{tabular}

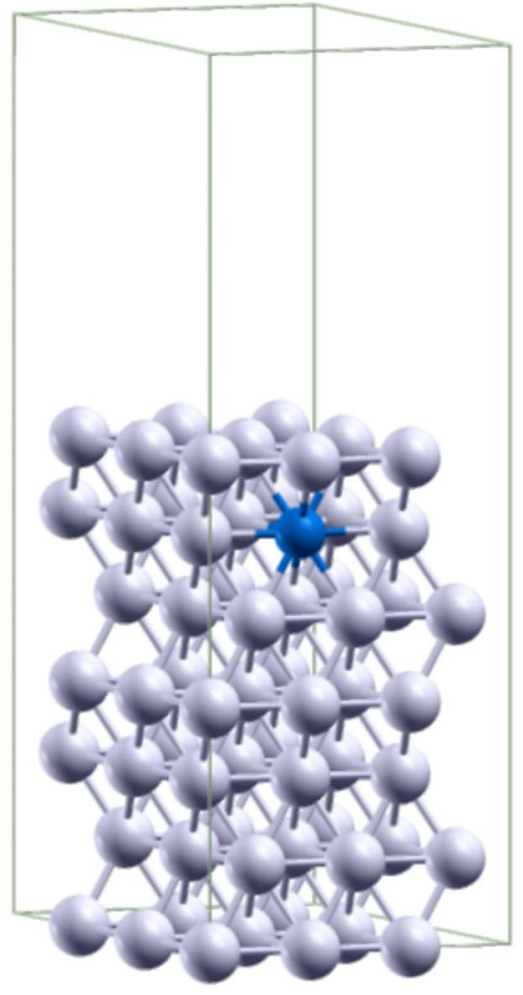

FIG. 1. (Color online) The $(3 \times 3)$ unit cell used for the study of segregation properties of $\mathrm{Cu}$ impurities in bulk Ag. The dark (blue) sphere represents a $\mathrm{Cu}$ atom.

surface energy. This is shown in Fig. 2 as the curve with black dots. The positive slope of the curve indicates that it is unfavorable for $\mathrm{Cu}$ impurities to migrate to the surface with respect to staying under the surface or in the bulk. ${ }^{38}$ The fact that the curve is very slightly convex (i.e., the black dots lie

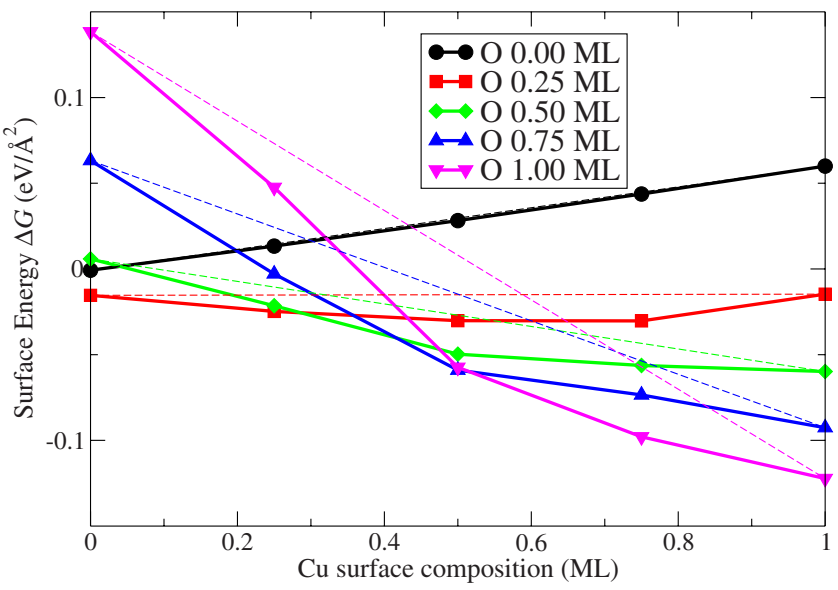

FIG. 2. (Color online) Change in Gibbs surface free energy at $T=0 \mathrm{~K}$ [cf. Eq. (6)] as a function of $\mathrm{Cu}$ concentration in the first layer of $\mathrm{Ag}(111)$. The oxygen coverages considered are indicated in the legend in monolayers (ML). The energy zero corresponds the surface energy of the clean $\operatorname{Ag}(111)$ surface. The straight dashed lines are obtained by joining the points corresponding to pure $\mathrm{Ag}$ and pure $\mathrm{Cu}$ in the first layer. 
below the straight line joining the two ends at composition 0 and 1), on the other hand, indicates that alloying in the first layer is slightly favorable with respect to phase separation into two structures that have on the first layer pure $\mathrm{Ag}$ and pure $\mathrm{Cu}$. We note that on the scale of Fig. 2, this is difficult to see, but is evident when plotted on a smaller scale. This behavior (slightly convex curve) is what has been found in the theoretical work by Christensen et al. ${ }^{38}$ for the $\mathrm{Ag}-\mathrm{Cu}$ surface alloy on the $\mathrm{Cu}(100)$ surface, employing the linear muffin-tin orbitals method in the tight-binding representation using the atomic-sphere approximation and the coherentpotential approximation and the local-density approximation for the exchange and correlation. ${ }^{38}$

The small tendency to alloy and the large difference in surface energy between $\operatorname{Ag}(111)$ and $\mathrm{Cu}(111)$ have dramatic structural effects as has been recently shown from UHV experiments in which $\mathrm{Cu}$ thin-film growth on $\mathrm{Ag}(111)$ was studied. ${ }^{39,40}$ It was found that at room temperature, upon deposition of $\mathrm{Cu}$ on $\mathrm{Ag}(111), \mathrm{Cu}$ forms islands that are encapsulated by one monolayer of $\mathrm{Ag}$, in agreement with our DFT results presented in this work (cf. Table II).

When oxygen is introduced into the picture, the situation changes completely: We find that the presence of oxygen chemisorbed on the alloy surface has the remarkable effect of reversing the slope of the (black dots) curve for the surface energy versus $\mathrm{Cu}$ surface composition, shown in Fig. 2. For these calculations, we consider a $\mathrm{Ag}-\mathrm{Cu}$ alloy in the first layer of the Ag slab, with oxygen adsorbed in the fcc site, i.e. in the most favorable adsorption site. Out of the four possible fcc sites in the $(2 \times 2)$ cell, we always select the ones next to the copper atoms present on the first layer for the oxygen adsorption. Here, we modeled the surface with a seven layer slab with alloy structures created on both sides of the slab. By fully relaxing the structure, we calculate the change in surface free energy at $T=0 \mathrm{~K}$ as a function of the $\mathrm{Cu}$ content in the first layer and the oxygen coverage. The results are shown in Fig. 2. We can see that the presence of at least a quarter of a monolayer (ML) of oxygen induces $\mathrm{Cu}$ to segregate to the surface, i.e., changes the slope of the curve from positive [for an oxygen coverage relative to the underlying $\mathrm{Ag}(111)$ lattice $\theta_{\mathrm{O}}<0.25 \mathrm{ML}$ ] to negative (for $\theta_{\mathrm{O}} \geqslant 0.25$ ML). The driving mechanism here is the strong affinity between oxygen and copper, which more than compensates the unfavorable surface energy of $\mathrm{Cu}$ with respect to $\mathrm{Ag}$ : The adsorption energies [cf. Eq. (1)] of oxygen at 0.25 ML coverage on $\operatorname{Ag}(111)$ and $\mathrm{Cu}(111)$ are 0.38 and $1.57 \mathrm{eV} /$ atom, respectively. We also see that in the absence of copper (i.e., the left end of the graph in Fig. 2), the most stable structure is the one with $\theta_{\mathrm{O}}=0.25 \mathrm{ML}$. At higher oxygen coverages, the oxygen-oxygen repulsion overcomes the formation of $\mathrm{O}-\mathrm{Ag}$ bonds, leading to an increase in surface energy with the oxygen coverage. At the opposite end of the graph, where a full monolayer of copper is present in the first layer of the slab, the surface energy decreases with the oxygen coverage: This is due to the contribution to the surface energy of the formation of strong $\mathrm{O}-\mathrm{Cu}$ bonds.

\section{B. Surface alloy and surface oxidelike structures}

Having seen that oxygen induces copper to segregate to the surface, we now consider various surface structures with different contents of copper and oxygen in the first layer. These structures, formed on top of a pure silver slab, are periodic, and the largest surface unit cell is the $(4 \times 4)$. As we will see in the next section, by changing the oxygen chemical potential according to Eq. (3), we investigate the stability of these surfaces by evaluating the surface free energy at various temperatures and pressures.

We consider three types of structures: (i) chemisorbed oxygen on the $\mathrm{Ag}-\mathrm{Cu}$ alloy in the first layer of the Ag slab (as described in the previous section), (ii) structures derived from copper(I) oxide $\mathrm{Cu}_{2} \mathrm{O}$, whose structure can be visualized as trilayers of $\mathrm{O}-\mathrm{Cu}-\mathrm{O}$ piled up on top of each other, and (iii) structures derived from copper(II) oxide $\mathrm{CuO}$.

The first set of structures is the ones considered in Fig. 2, and we label them OxML/CuyML, where $x$ and $y$ are the content of $\mathrm{O}$ and $\mathrm{Cu}$, expressed in monolayers with respect to the underlying $\operatorname{Ag}(111)$ surface. Some of these structures, in particular, the ones with high $\mathrm{Cu}$ content, involve large atomic rearrangements that are due to the large $\mathrm{Cu}-\mathrm{Cu}$ distances induced by the underlying $\operatorname{Ag}(111)$ lattice. As an example, we consider two structures, one at low oxygen coverage $(\mathrm{O} 0.25 \mathrm{ML} / \mathrm{Cu} 1 \mathrm{ML})$ and one at high oxygen coverage (O1 ML/Cu1 ML). In the first case, we find that oxygen adsorbs at a very reduced height in the fcc hollow site of the (111) surface $[0.43 \AA$ compared to $1.22 \AA$ for the $\mathrm{O} / \mathrm{Cu}(111)$ system at the same coverage]. In the second case, half the oxygen atoms penetrate below the copper layer, leading to a trilayerlike structure with a $(2 \times 1)$ periodicity. In this structure half the oxygen atoms are positioned $0.95 \AA$ above the average plane of $\mathrm{Cu}$ atoms and half $0.86 \AA$ below it. The atomic geometry is illustrated in Fig. 3(d).

For the second set of $\mathrm{Cu}_{2} \mathrm{O}$-like structures, we use the label $p 2$ or $p 4$ depending on whether the periodicity of the structure is $(2 \times 2)$ or $(4 \times 4)$ with respect to the clean $\mathrm{Ag}$ surface. The atomic geometry of the $p 2$ structure is shown in Fig. 3(a). Here, each $\mathrm{Cu}$ is linearly bonded with two $\mathrm{O}$ and each $\mathrm{O}$ is bonded to three $\mathrm{Cu}$ in a ringlike structure similar to the ones proposed for thin oxidelike layers on $\mathrm{Ag}(111)$ (Ref. $3)$ and $\mathrm{Cu}(111){ }^{41}$ The $p 4-\mathrm{OCu}_{3}$ is a $p 4$ structure in which an $\mathrm{OCu}_{3}$ unit has been removed [see Fig. 3(b)]. In $p 4+\mathrm{Cu}$ and $p 4+\mathrm{O}, \mathrm{a} \mathrm{Cu}$ atom and an $\mathrm{O}$ atom, respectively, have been added in an fcc adsorption site. " $\mathrm{Cu}_{6}$ " is analogous to the structure derived from the recently proposed " $\mathrm{Ag}_{6}$ " structure for the $(4 \times 4)-\mathrm{O} / \mathrm{Ag}(111)$ system. ${ }^{42,43}$ We note that constraining an $\mathrm{O}-\mathrm{Cu}-\mathrm{O}$ trilayer of $\mathrm{Cu}_{2} \mathrm{O}(111)$ to be commensurate with the $p 2$ (or equivalently a $p 4$ ) periodicity of $\mathrm{Ag}(111)$ results in a compression (7\%) of the trilayer with respect to its equilibrium lattice constant in bulk copper oxide. We also consider thicker films of $\mathrm{Cu}_{2} \mathrm{O}$ (up to five atomic layers) in order to extrapolate the behavior of bulk $\mathrm{Cu}_{2} \mathrm{O}$. As we will show in the next section, the two most relevant thin oxidelike structures are $p 2$ and $p 4-\mathrm{OCu}_{3}$. For these $\mathrm{Cu}_{2} \mathrm{O}$-like configurations, the average binding energies per oxygen atom are 1.41 and $1.37 \mathrm{eV}$, respectively, and the $\mathrm{Cu}-\mathrm{O}$ bond lengths are between 1.84 and $1.85 \AA$ in both cases. For comparison, in bulk $\mathrm{Cu}_{2} \mathrm{O}$, the computed formation energy per oxygen atom is $1.26 \mathrm{eV}$ [experimental value of $1.75 \mathrm{eV}$ (Ref. 44)] and the $\mathrm{Cu}-\mathrm{O}$ bond length is $1.88 \AA$.

For the third set of structures, we consider thin layers of CuO-like structures in a $(2 \times 2)$ cell. Forcing the oxide layer 

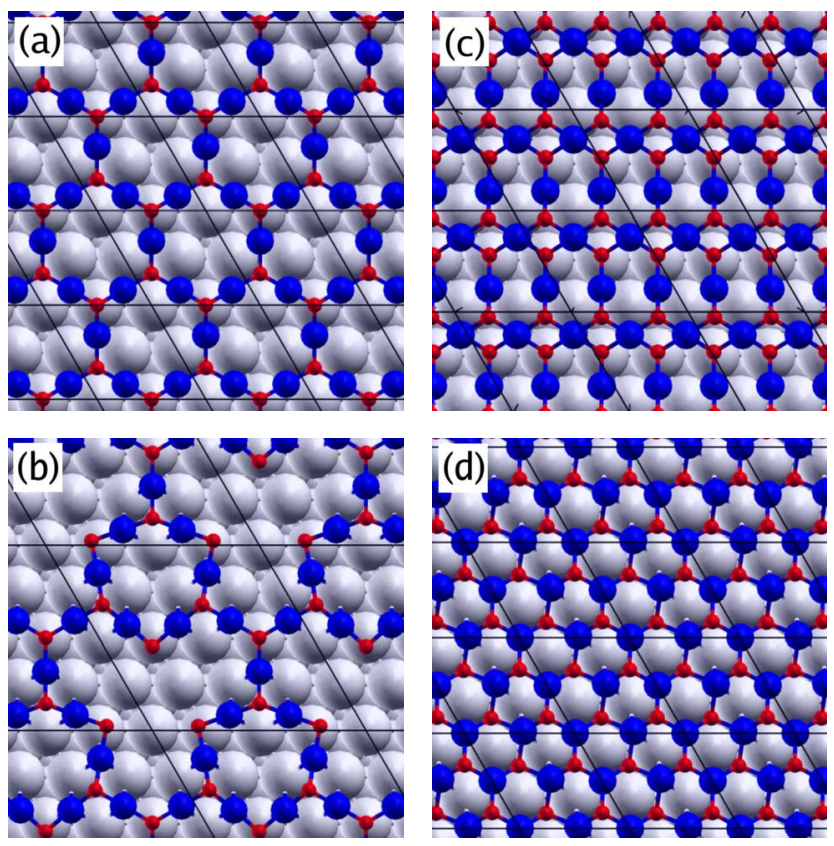

FIG. 3. (Color online) Top view of four surface structures considered: (a) "p2," (b) "p4-OCu 3 ," (c) "CuO(1L)," and (d) "O1ML/ Cu1ML" (see text). The gray spheres represent the underlying $\operatorname{Ag}(111)$ substrate. Copper atoms are shown as large dark (blue) circles and oxygen atoms are the small dark (red) circles. The black lines represent the surface unit cells.

to match the $(2 \times 2)$ lattice of the underlying $\operatorname{Ag}(111)$ surface leads to a compression of the $\mathrm{Cu}-\mathrm{O}$ bond length of about $3 \%$ with respect to the bulk $\mathrm{CuO}$ value. For the one layer $\mathrm{CuO}$ structure [labeled $\mathrm{CuO}(1 \mathrm{~L})]$, shown in Fig. 3(c)], we find that the $\mathrm{Cu}-\mathrm{O}$ bond length in the square planar pattern is $1.91 \AA$ and the binding energy per oxygen atom is $1.16 \mathrm{eV}$. We consider a number of possible defective $\mathrm{CuO}$-like thin layer structures (not shown here), but we find them not to be stable. We then consider thicker $\mathrm{CuO}$-like films (up to five atomic layers) in order to extrapolate the behavior of bulk $\mathrm{CuO}$. In this case, we must bear in mind that bulk $\mathrm{CuO}$ is poorly described with DFT-PBE: $\mathrm{CuO}$ is a strongly correlated antiferromagnetic semiconductor, with a monoclinic structure $\left(a=4.65 \AA, b=3.41 \AA, c=5.11 \AA\right.$, and $\left.\beta=99.5^{\circ}\right)$, where $\mathrm{Cu}$ is linearly bonded to two $\mathrm{O}$ atoms and $\mathrm{O}$ is bound to two $\mathrm{Cu}$ atoms. ${ }^{45}$ DFT-PBE, on the other hand, predicts $\mathrm{CuO}$ to be a metal with an almost orthorthorombic structure $\left(a=4.34 \AA, b=4.01 \AA, c=5.22 \AA\right.$, and $\left.\beta=92.2^{\circ}\right)$, in which each $\mathrm{O}$ is tetrahedrally coordinated to four $\mathrm{Cu}$ and each $\mathrm{Cu}$ is bonded in a square planar geometry to four $\mathrm{O}$ atoms. The predictions for thick films of $\mathrm{CuO}$ must therefore be regarded as qualitative. The computed formation energy per oxygen atom in bulk $\mathrm{CuO}$ is $1.23 \mathrm{eV}$ [experimental value of $1.63 \mathrm{eV}$ (Ref. 44)] and the $\mathrm{Cu}-\mathrm{O}$ bond length is about $1.97 \AA$ (experimental value of $1.67 \AA$ ). Going beyond the DFT-PBE description is a major task. Calculations for $\mathrm{CuO}$ and $\mathrm{Cu}_{2} \mathrm{O}$ using GGA $+U$ plus $G_{0} W_{0}$ (Ref. 46) as well as other methodologies ${ }^{47}$ are in progress.

In addition to the structures considered in this work, it is likely that other structures involving thin oxidelike layers with similar surface energies exist for this system. Recent
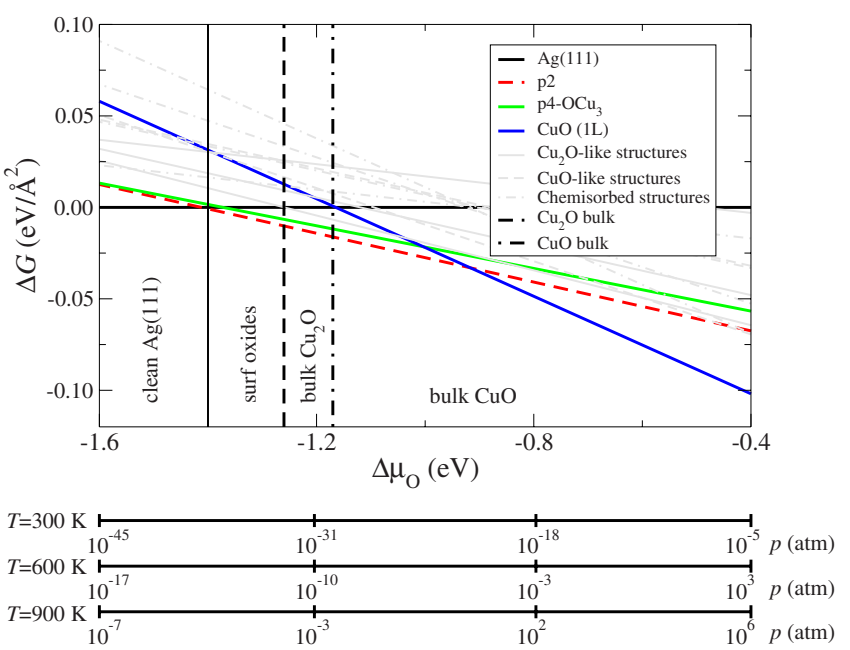

FIG. 4. (Color online) Change in Gibbs surface free energy as a function of the change in oxygen chemical potential $\Delta \mu_{\mathrm{O}}$. The chemical potential of oxygen is measured with respect to half the total energy of the free $\mathrm{O}_{2}$ molecule. The vertical dashed lines separate the regions of stability of the clean $\mathrm{Ag}(111)$ surface, the surface oxides, and bulk oxides $\mathrm{Cu}_{2} \mathrm{O}$ and $\mathrm{CuO}$.

works on $\mathrm{O} / \mathrm{Ag}(111)$ (Refs. 3, 42, and 43) and $\mathrm{O} / \mathrm{Pd}(111)$ (Ref. 48) have shown that for these systems a multitude of structures with similar energetics can be found.

\section{Thermodynamic diagram of the $\mathrm{O} / \mathrm{Cu} / \mathrm{Ag}(111)$ system}

In Fig. 4, we report the change in Gibbs surface free energy of the structures considered as a function of the change in oxygen chemical potential. We also show at the bottom of the plot, for three values of temperature $(300,600$, and $900 \mathrm{~K}$ ), the value of pressure corresponding to the chemical potential shown in the abscissa of the plot.

As we can see from Eq. (6), the slope of the lines in Fig. 4 is proportional to the oxygen coverage, i.e., the higher the oxygen content, the steeper the line. The vertical dashed line at $\Delta \mu_{\mathrm{O}}=-1.26 \mathrm{eV}$ represents the change in chemical potential above which $\mathrm{Cu}$ oxidizes to $\mathrm{Cu}_{2} \mathrm{O}$, which, as one can show, corresponds to the computed heat of formation of $\mathrm{Cu}_{2} \mathrm{O}$. The vertical dashed line at $\Delta \mu_{\mathrm{O}}=-1.23 \mathrm{eV}$, on the other hand, represents the change in chemical potential above which $\mathrm{Cu}$ oxidizes to $\mathrm{CuO}$. We can therefore see that in the range of values of the oxygen chemical potential considered $\left(-1.6<\Delta \mu_{\mathrm{O}}<-0.4 \mathrm{eV}\right)$, the thermodynamically stable structures are pure $\mathrm{Ag}$ for $\Delta \mu_{\mathrm{O}}<-1.43 \mathrm{eV}, p 2$ and $p 4-\mathrm{OCu}_{3}$ (almost degenerate) between $-1.43<\Delta \mu_{\mathrm{O}}<-1.26 \mathrm{eV}$, bulk copper(I) oxide $\mathrm{Cu}_{2} \mathrm{O}$ for $-1.26<\Delta \mu_{\mathrm{O}}<-1.23 \mathrm{eV}$, and bulk copper(II) oxide $\mathrm{CuO}$ for $\Delta \mu_{\mathrm{O}}>-1.23 \mathrm{eV}$. It is interesting to note that none of the alloyed chemisorbed structures (the structures labeled as $\mathrm{O} x \mathrm{ML} / \mathrm{Cu} y \mathrm{ML})$ are thermodynamically stable in this range of chemical potential, while there is a small region in which two-dimensional surface oxides are stable. This situation is similar to what has been found for the $\mathrm{O} / \mathrm{Cu}(111)$ system, ${ }^{41}$ where it was argued that copper oxidation does not proceed via ordered chemisorbed structures at variance with other 
transition metal structures. We also find that none of the $\mathrm{O} / \mathrm{Ag}$ structures, containing no $\mathrm{Cu}$ (not shown in Fig. 4), are thermodynamically stable in the range considered here. This is not unexpected, given the weaker strength of the $\mathrm{Ag}-\mathrm{O}$ bond with respect to the $\mathrm{Cu}-\mathrm{O}$ one.

\section{Modeling systems of known Cu surface content}

Having identified, among those considered here, the thermodynamically most stable ordered structures on an infinite $\operatorname{Ag}(111)$ surface, we now consider the situation in which we have a well defined $\mathrm{Cu}$ surface content. For a finite system (e.g., a Ag nanoparticle) of known dimensions and well defined $\mathrm{Cu}$ content, if we assume that all the $\mathrm{Cu}$ present in a spherical nanoparticle segregates to the surface in a site of the Ag fcc lattice, given the size of nanoparticle and the total $\mathrm{Cu}$ content, we can estimate the $\mathrm{Cu}$ surface content. For example, in a $50 \mathrm{~nm}$ spherical nanoparticle with a $1 \% \mathrm{Cu}$ content (typical values of nanoparticle size and copper content of $\mathrm{Ag}-\mathrm{Cu}$ alloys used in experiments in which the effect of $\mathrm{Cu}$ impurities was investigated ${ }^{12}$ ), the (average) surface $\mathrm{Cu}$ content is $0.35 \mathrm{ML}$, where $1 \mathrm{ML}$ corresponds to the first layer of the nanoparticle being pure copper. Experiments carried out on $\mathrm{Ag}-\mathrm{Cu}$ alloys with $0.1 \%-1 \% \mathrm{Cu}$ content at $\sim 500 \mathrm{~K}$ have shown that in an oxidizing environment the $\mathrm{Cu}$ surface content is in the range 0.1-0.75 ML. ${ }^{12}$

Given the fact that $\operatorname{Ag}(111)$ is the lowest energy surface for silver, ${ }^{49}$ the typical understanding is that a large portion of the nanoparticle surface will consist of (111) planes. This is not necessarily true at high temperature, where the particle can be quite round, especially in the case of noble metals, and in the presence of oxidelike structures on the surface, that can alter the relative surface energies compared to the case of pure Ag at zero temperature. We will, however, restrict ourselves to this particular surface and use the results shown in Fig. 4 to predict which structures will be present on the surface as a function of the copper content and the oxygen chemical potential. We also exploit the vast literature available for the $\mathrm{O} / \mathrm{Ag}$ system $^{3,42}$ to include the most stable structures for the system in the absence of copper.

As an example, we show explicitly the case for $\Delta \mu_{\mathrm{O}}=-0.80 \mathrm{eV}$, corresponding to an oxygen pressure of $10^{-3} \mathrm{~atm}$ at the temperature of $600 \mathrm{~K}$. Figure 5 shows the change in surface free energy at $\Delta \mu_{\mathrm{O}}=-0.80 \mathrm{eV}$ of all the structures we have considered (a total of 55 structures, including the $\mathrm{O} / \mathrm{Ag}$ ones) as a function of the $\mathrm{Cu}$ surface content. By constructing the convex hull, i.e., the curve obtained by joining those structures that are stable with respect to linear combinations of structures at other compositions that would yield the same total composition, we identify the structures (indicated by the red dots in Fig. 5) that are stable against phase separation into any two other structures. The structures belonging to the convex hull, for this particular value of chemical potential, are "Ag" [clean $\mathrm{Ag}(111)$ surface], $p 4-\mathrm{Cu}_{3} \mathrm{O}, p 2$, and $\mathrm{CuO}$ (b) (bulk $\mathrm{CuO}$ ). Although in Fig. 5 we show only the portion of the diagram up to a $\mathrm{Cu}$ content of $1 \mathrm{ML}$, we have computed structures including up to five $\mathrm{CuO}$ layers in order to be able to extrapolate the behavior of bulk $\mathrm{CuO}$.

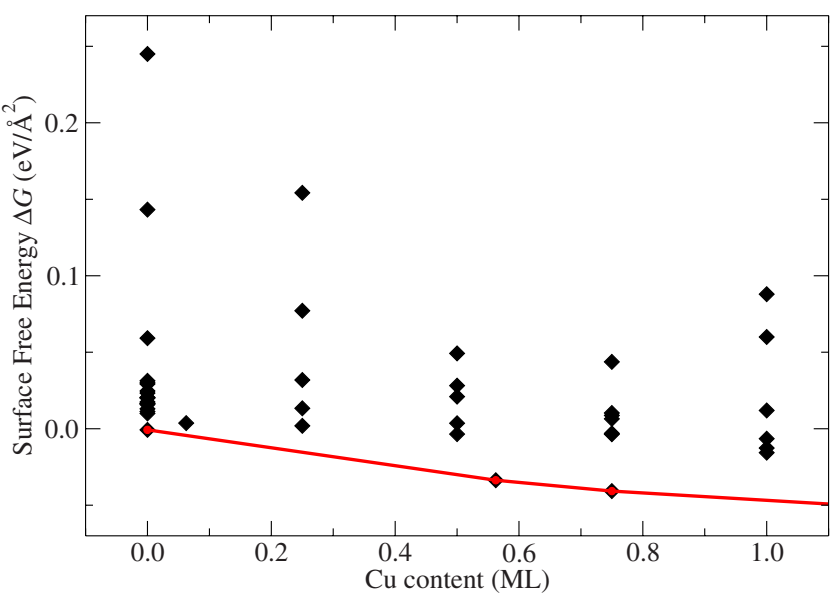

FIG. 5. (Color online) Construction of the convex hull for the case of $\Delta \mu_{\mathrm{O}}=-0.80 \mathrm{eV}$. The energy zero corresponds to the clean $\operatorname{Ag}(111)$ surface.

For a specific $\mathrm{Cu}$ content, e.g., $0.25 \mathrm{ML}$, the convex hull plot helps to predict that a mixture of pure $\mathrm{Ag}$ and $p 4-\mathrm{Cu}_{3} \mathrm{O}$ (rather than a single ordered structure with $0.25 \mathrm{ML} \mathrm{Cu}$ content) will be present on the $\operatorname{Ag}(111)$ surface at $\Delta \mu_{\mathrm{O}}=-0.80 \mathrm{eV}$ in a ratio given by the lever rule, i.e., by the mass conservation law. By repeating this scheme for all the values of the oxygen chemical potential in the range considered in this work, we build a phase diagram as a function of the oxygen chemical potential and the copper surface content. This is shown in Fig. 6. We find that the convex hull, for high $\mathrm{Cu}$ content, always includes the structure with the largest number of $\mathrm{CuO}$ layers (or $\mathrm{Cu}_{2} \mathrm{O}$ layers at lower oxygen chemical potential), i.e., bulk oxide formation is favored beyond a certain $\mathrm{Cu}$ surface content. We therefore cut the plot in Fig. 6 at a $\mathrm{Cu}$ composition of $1.50 \mathrm{ML}$, where it is understood that the rightmost structure is "bulk" oxide. However, care must be taken in the meaning given to such bulk struc-

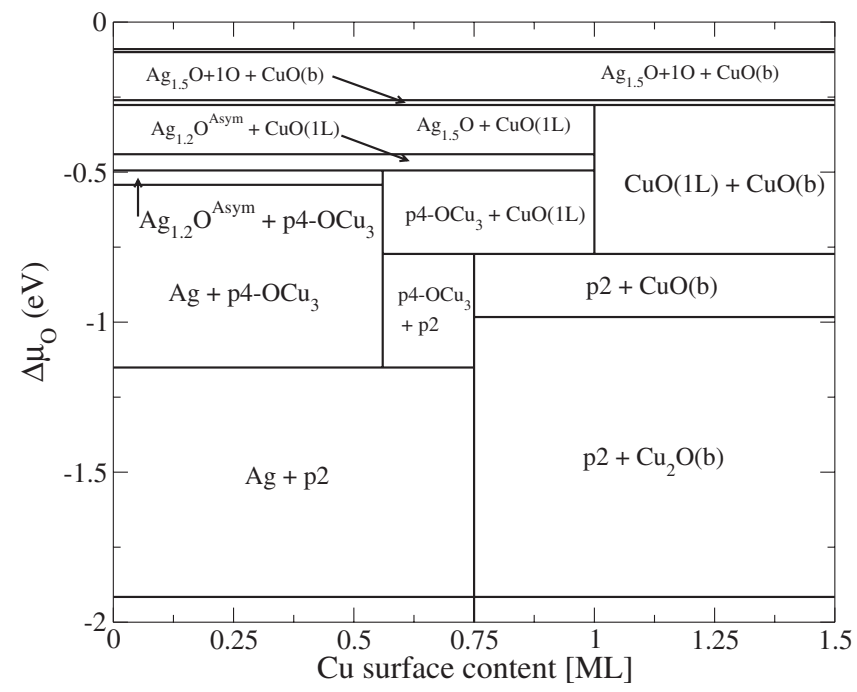

FIG. 6. Surface phase diagram showing the structures belonging to the convex hull as a function of the $\mathrm{Cu}$ surface content and the change in oxygen chemical potential. 
ture in the context of a finite system as the one we are modeling here. We interpret the numerical evidence of the presence of the bulk structure in the convex hull as a tendency for the formation of thick patches of either $\mathrm{CuO}$ or $\mathrm{Cu}_{2} \mathrm{O}$.

In the phase diagram shown in Fig. 6, we have explicitly written the two structures coexisting in a number of regions around the values of interest of oxygen chemical potential and copper content. The labels $\mathrm{Ag}_{1.5} \mathrm{O}$ and $\mathrm{Ag}_{1.2} \mathrm{O}^{\text {Asym }}$ refer to $\mathrm{O} / \mathrm{Ag}$ structures identified in Ref. 3. If we focus on the region around the chemical potential of interest in typical industrial applications $(p=1 \mathrm{~atm}, T=600 \mathrm{~K}$, corresponding to $\Delta \mu_{\mathrm{O}} \sim-0.61 \mathrm{eV}$ ) and for contents of copper below half a monolayer, we predict patches of one layer oxidic structures $\left(p 4-\mathrm{Cu}_{3} \mathrm{O}\right)$ to coexist with the clean $\mathrm{Ag}$ surface. At higher values of oxygen chemical potential, on the other hand, $\mathrm{O} / \mathrm{Ag}$ structures can be found in coexistence with the $p 4-\mathrm{Cu}_{3} \mathrm{O}$ structure. For higher copper contents, the "CuO(1L)" and the "p2" structures are present in the phase diagram above and below $\Delta \mu_{\mathrm{O}} \sim-0.75 \mathrm{eV}$, respectively. Finally, for even higher copper contents, bulk $\mathrm{CuO}$ is predicted to form.

This picture is consistent with recent experiments performed on the $\mathrm{Ag}-\mathrm{Cu}$ system under catalytic conditions. ${ }^{50} \mathrm{In}$ these experiments $\mathrm{Ag}-\mathrm{Cu}$ nanopowders $(\sim 100 \mathrm{~nm}$ in diameter and $2.5 \% \mathrm{Cu}$ ) are used as the catalyst for ethylene epoxidation at $T=520 \mathrm{~K}$ and $p=0.5 \mathrm{mbar}$. Through a combination of in situ XPS and near edge X-ray absorption fine structure measurements, thin layers of $\mathrm{CuO}$ are found be to present on the surface, while no signs on a surface alloy are detected. Areas of clean Ag are also present on the surface, in agreement with our theoretical calculations.

These results show that the simple structure adopted in Ref. 11 for the Ag-Cu surface alloy to model theoretically the ethylene epoxidation reaction is not stable and is significantly different from what we expect to be relevant under high pressure conditions. The model used in Ref. 11 assumes a $(2 \times 2)$ periodicity for the $\operatorname{Ag}(111)$ surface in which one atom out of four is replaced with copper, and oxygen is chemisorbed on it. Our results suggest on the other hand that a model that includes, depending on the $\mathrm{Cu}$ surface content, clean $\operatorname{Ag}(111)$, patches of oxidelike thin layers and thick layers of $\mathrm{CuO}$ is a more appropriate model for the $\mathrm{Ag}-\mathrm{Cu}$ catalyst (111) surface.

\section{CONCLUSIONS}

In summary, through density functional theory calculations and employing ab initio atomistic thermodynamics to include the effects of pressure and temperature, we have investigated the (111) surface of the $\mathrm{Ag}-\mathrm{Cu}$ alloy. We have shown that, in the absence of oxygen, copper impurities in silver prefer to stay directly below the silver surface, rather than in the bulk or on the surface. The presence of oxygen, on the other hand, has the effect to induce copper to segregate to the surface due to the strength of the $\mathrm{O}-\mathrm{Cu}$ bond relative to the $\mathrm{O}-\mathrm{Ag}$ one. Both findings are in agreement with experimental evidence. We have investigated structures involving chemisorbed oxygen on the alloy surface, as well as oxidelike structures on the $\operatorname{Ag}(111)$ surface. Through the construction of the oxygen chemical potential dependent convex hull, we have been able to identify, as a function of the surface copper content, the combinations of structures that are most stable at that particular temperature and pressure. In the region of interest, our results suggest that, depending on the copper surface content, clean $\operatorname{Ag}(111)$ and thin copper oxidelike structures $\left[p 4-\mathrm{Cu}_{3} \mathrm{O}, p 2\right.$, and $\mathrm{CuO}(1 \mathrm{~L})]$ can coexist. This model for the (111) surface of the $\mathrm{Ag}-\mathrm{Cu}$ catalyst differs substantially from the structures used in earlier works to investigate the effect of copper impurities in silver on the mechanism of ethylene epoxidation. Our results suggest that to gain insight into the full catalytic cycle, one should consider oxygen species such as those in the proposed structures, rather than just oxygen chemisorbed on the the clean alloy surface. We envisage that the theoretical methodology reported in the present paper will also be useful for characterizing other alloy catalysts under realistic conditions.
${ }^{1}$ R. A. van Santen and H. P. C. E. Kuipers, Adv. Catal. 35, 265 (1987)

${ }^{2}$ W.-X. Li, C. Stampfl, and M. Scheffler, Phys. Rev. Lett. 90, 256102 (2003).

${ }^{3}$ A. Michaelides, K. Reuter, and M. Scheffler, J. Vac. Sci. Technol. A 23, 1487 (2005).

${ }^{4}$ V. I. Bukhtiyarov, M. Hävecker, V. V. Kaichev, A. Knop-Gericke, R. W. Mayer, and R. Schlögl, Phys. Rev. B 67, 235422 (2003).

${ }^{5}$ K. Reuter, D. Frenkel, and M. Scheffler, Phys. Rev. Lett. 93, 116105 (2004).

${ }^{6}$ J. Rogal, K. Reuter, and M. Scheffler, Phys. Rev. Lett. 98, 046101 (2007).

${ }^{7}$ B. L. M. Hendriksen, S. C. Bobaru, and J. W. M. Frenkel, Top. Catal. 36, 43 (2005).

${ }^{8}$ K. Reuter and M. Scheffler, Phys. Rev. Lett. 90, 046103 (2003).

${ }^{9}$ S. Linic and M. A. Barteau, J. Am. Chem. Soc. 124, 310 (2002).
${ }^{10}$ S. Linic and M. A. Barteau, J. Am. Chem. Soc. 125, 4034 (2003).

${ }^{11}$ S. Linic, J. Jankowiak, and M. A. Barteau, J. Catal. 224, 489 (2004).

${ }^{12}$ J. T. Jankowiak and M. A. Barteau, J. Catal. 236, 366 (2005).

${ }^{13}$ J. H. Sinfelt, Bimetallic Catalysts: Discoveries, Concepts and Application (Wiley, New York, 1983).

${ }^{14}$ P. Liu and J. K. Nørskov, Phys. Chem. Chem. Phys. 3, 3814 (2001).

${ }^{15}$ J. Greeley and M. Mavrikakis, Nat. Mater. 3, 810 (2004).

${ }^{16}$ J. Greeley, T. F. Jaramillo, J. Bonde, I. Chorkendorff, and J. Nørskov, Nat. Mater. 5, 909 (2006).

${ }^{17}$ R. D. Schmidt-Whitley, M. Martinez-Clemente, and A. Revcolevschi, J. Cryst. Growth 23, 113 (1974).

${ }^{18}$ J. P. Perdew, K. Burke, and M. Ernzerhof, Phys. Rev. Lett. 77, 3865 (1996).

${ }^{19}$ D. Vanderbilt, Phys. Rev. B 41, 7892 (1990). 
${ }^{20}$ The ultrasoft pseudopotentials for $\mathrm{Ag}, \mathrm{Cu}$, and $\mathrm{O}$ were taken from the PWSCF pseudopotential download page: http:// www.pwscf.org/pseudo/htm (files: Ag.pbe-d-rrkjus.UPF, Cu.pbe-d-rrkjus.UPF, and O.pbe-rrkjus.UPF).

${ }^{21}$ H. J. Monkhorst and J. D. Pack, Phys. Rev. B 13, 5188 (1976).

${ }^{22}$ N. Marzari, D. Vanderbilt, A. De Vita, and M. C. Payne, Phys. Rev. Lett. 82, 3296 (1999).

${ }^{23}$ The value of $16 \mathrm{meV}$ is obtained comparing the adsorption energies using the $19 \times 19 \times 1$ and $30 \times 30 \times 1$ meshes.

${ }^{24}$ S. Baroni, A. D. Corso, S. de Gironcoli, P. Giannozzi, C. Cavazzoni, G. Ballabio, S. Scandolo, G. Chiarotti, P. Focher, A. Pasquarello et al., QUANTUM-ESPRESSO (http://www.pwscf.org).

${ }^{25}$ We checked the effect of increasing the thickness of the slab. In the case of the chemisorbed structures, going from 7 to 11 layer slabs leads to a change of the surface energy of the order of $2 \mathrm{meV} / \AA^{2}$. In the oxidelike structures, going from 3 to 5 layer slabs amounts to a change of the surface energy of the order of $1 \mathrm{meV} / \AA^{2}$.

${ }^{26}$ Increasing the vacuum thickness from $12 \AA$ to $17 \AA$ changes the adsorption energy of an oxygen atom on $\mathrm{Ag}(111)$ by $11 \mathrm{meV}$, i.e., from 1.021 to $1.032 \mathrm{eV}$.

${ }^{27}$ N. W. Ashcroft and N. D. Mermin, Solid State Physics (Saunders College, New York, 1976).

${ }^{28}$ K. P. Huber and G. Herzberg, Molecular Spectra and Molecular Structure IV: Constants of Diatomic Molecules (Van Nostrand Reinhold, New York, 1979).

${ }^{29}$ C. Stampfl, Catal. Today 105, 17 (2005).

${ }^{30}$ K. Reuter and M. Scheffler, Phys. Rev. B 65, 035406 (2001).

${ }^{31}$ W.-X. Li, C. Stampfl, and M. Scheffler, Phys. Rev. B 67, 045408 (2003).

${ }^{32}$ K. Reuter and M. Scheffler, Phys. Rev. B 65, 035406 (2001).

${ }^{33}$ D. R. Stull and H. Prophet, JANAF Thermochemical Tables, 2nd ed. (U.S. National Bureau of Standards, Washington, D.C., 1971).

${ }^{34}$ F. Ducastelle, Order and Phase Stability in Alloys (NorthHolland, Amsterdam, 1991).

${ }^{35}$ M. Hansen, Consitution of Binary Alloys (McGraw-Hill, New
York, 1958).

${ }^{36}$ F. R. D. Boer, R. Boom, W. C. M. Mattens, A. R. Miederna, and A. K. Niessen, Cohesion in Metals (North-Holland, Amsterdam, 1988).

${ }^{37}$ S. A. Lindgren, L. Walldén, J. Rundgren, and P. Westrin, Phys. Rev. B 29, 576 (1984).

${ }^{38}$ A. Christensen, A. V. Ruban, P. Stoltze, K. W. Jacobsen, H. L. Skiver, J. K. Nørskov, and F. Besenbacher, Phys. Rev. B 56, 5822 (1997).

${ }^{39}$ C. Maurel, M. Abel, M. Koudia, F. Bocquet, and L. Porte, Surf. Sci. 596, 45 (2005).

${ }^{40}$ F. Bocquet, C. Maurel, J.-M. Roussel, M. Abel, M. Koudia, and L. Porte, Phys. Rev. B 71, 075405 (2005).

${ }^{41}$ A. Soon, M. Todorova, B. Delley, and C. Stampfl, Phys. Rev. B 73, 165424 (2006).

${ }^{42}$ J. Schnadt, A. Michaelides, J. Knudsen, R. T. Vang, K. Reuter, E. Laegsgaard, M. Scheffler, and F. Besenbacher, Phys. Rev. Lett. 96, 146101 (2006).

${ }^{43}$ M. Schmid, A. Reicho, A. Stierle, I. Costina, J. Klikovits, P. Kostelnik, O. Dubay, G. Kresse, J. Gustafson, E. Lundgren et al., Phys. Rev. Lett. 96, 146102 (2006).

${ }^{44}$ CRC Handbook of Chemistry and Physics (CRC, Boca Raton, FL, 1995).

${ }^{45}$ J. Y. Kim, J. A. Rodriguez, J. C. Hanson, A. I. Frenkel, and P. Lee, J. Am. Chem. Soc. 125, 10684 (2003).

${ }^{46}$ H. Jiang, R. Gomez-Abal, X. Li, P. Rinke, and M. Scheffler (unpublished).

${ }^{47}$ Q.-M. Hu, K. Reuter, and M. Scheffler, Phys. Rev. Lett. 98, 176103 (2007).

${ }^{48}$ J. Klikovits, E. Napetschnig, M. Schmid, N. Seriani, O. Dubay, G. Kresse, and P. Varga, Phys. Rev. B 76, 045405 (2007).

${ }^{49}$ L. Vitos, A. V. Ruban, H. L. Skriver, and J. Kollar, Surf. Sci. 411, 186 (1998).

${ }^{50}$ S. Zafeiratos, M. Hävecker, D. Teschner, E. Vass, P. Schnörch, F. Girgsdies, T. Hansen, A. Knop-Gericke, R. Schlögl, and V. Bukhtiyarov (unpublished). 